NOTAS 



\title{
ANÁLISIS PRELIMINAR DE CAMBIO CLIMÁTICO EN LA CIÉNAGA DE ZAPATA*
}

\author{
Bárbaro V. Moya \\ Centro Meteorológico Provincial. \\ Ministerio de Ciencia, Tecnología y Medio Ambiente (CITMA). Matanzas (Cuba) \\ Ángel Alfonso, Mario Gutiérrez, Nelvis Gómez y Mabel García \\ Unidad de Medio Ambiente. \\ Ministerio de Ciencia, Tecnología y Medio Ambiente (CITMA). Matanzas (Cuba)
}

\section{RESUMEN}

La Ciénaga de Zapata es el mayor humedal del Caribe insular. Es un humedal frágil y vulnerable al cambio climático. El presente trabajo hace un análisis preliminar de la relación de la Ciénaga de Zapata con el cambio climático.

El análisis muestra apuntes sociales y económicos que caracterizan al humedal. Se plantean algunos de los valores ecológicos de la ciénaga como parte de esta caracterización y a continuación se muestran vulnerabilidades del ecosistema ante el cambio climático, los impactos de este cambio en el humedal y se da una serie de medidas de adaptación, que culminan con la propuesta de un proyecto integral con una serie de salidas que permitan atenuar las incertidumbres y favorecer la explotación y manejo del humedal. En la propuesta de proyecto se incluye también el humedal de Majaguillar ubicado en la costa Norte de la provincia de Matanzas.

Palabras clave: Cambio climático, vulnerabilidad, impactos, adaptación.

\section{ABSTRACT}

The Cienaga de Zapata is the biggest wetland located in the islands of the Caribbean sea. It is a fragile and vulnerable to climate change wetland. The current study develops a preliminary analyse of the relationship existing between Cienaga de Zapata and the climate change.

* El trabajo forma parte de una conferencia impartida en el Simposio Internacional Humedales 2003. Ciénaga de Zapata. Matanzas. Cuba. 
The analyse shows economic and social notes which characterise the wetland. It also shows some ecological values of the wetland and vulnerabilities of the ecosystem to climate change, the impacts of mentioned change in the wetland and give some measures to ensure the adaptation for the future climate change scenario. At the end, the study proposes an integral project with objectives ways to improve the exploitation and management of the wetland, develop an adaptation program and reduce the uncertainties. The Majaguillar wetland, located in the north coast of Matanzas province is also included in this project.

Key words: Climate change, vulnerability, impacts, adaptation.

\section{INTRODUCCIÓN}

Desde comienzos de la década de los noventa, en que ocurrieron ocho de los diez años más calientes del pasado siglo, el hombre ha ido tomando conciencia de la influencia de sus actos en el aumento de la concentración de gases en la atmósfera. Estos gases tienen disímiles efecto, en algunos casos aumentan el potencial de invernadero de la atmósfera, en otros atentan contra la formación del ozono estratosférico, que constituye la sombrilla que protege la tierra de los rayos ultravioletas del sol, en otros provocan deposiciones ácidas secas y húmedas, provocando afectaciones a la flora, a la fauna y a la actividad microbiana llegando a provocar la destrucción tanto de ecosistemas terrestres, como marinos, y en otros casos estos gases tienen un efecto nocivo sobre la salud humana, llegando en algunos casos, no sólo aumentar la morbilidad, sino a favorecer crecimiento en la mortalidad.

Estos problemas no se circunscriben a zonas o localidades específicas, sino que tienen repercusión desde la escala local hasta la global. Cuba, no es una excepción y ya se vienen detectando, mediante el sistema de vigilancia del clima que desarrolla el Instituto de Meteorología a nivel nacional, y su red de centros provinciales, una serie de variaciones en el clima, no demuestra que este ocurriendo un cambio climático, si da un alerta de que existe una variación en el comportamiento del clima respecto a las últimas décadas.

Teniendo en cuenta lo anterior y el citado sistema de vigilancia que incluye también la vigilancia meteorológica, y de la calidad del aire, se han desarrollado en la actualidad una serie de estudios dirigidos a determinar las incidencias del cambio climático y los efectos que pueden tener sobre el país el resto de los principales problemas atmosféricos. Una parte de estos estudios esta dirigida a los impactos y medidas de adaptación al llamado cambio climático y su vinculación con la esfera socioeconómica en diferentes territorios de la provincia de Matanzas.

En el caso de la relación del cambio climático con los humedales apenas ha sido abordada, no obstante algunos estudios preliminares se han desarrollado. En este caso se dirigen esfuerzos a dar un primer acercamiento a la relación del humedal Ciénaga de Zapata con el cambio climático y a emitir algunas medidas de adaptación que favorezca la mitigación de los impactos y la adaptación a los escenarios climáticos futuros.

\section{DESARROLLO}

La Ciénaga de Zapata es el mayor humedal del Caribe insular y el más importante de Cuba. Es reserva de la biosfera desde al año 1999 y sitio Ramsar desde el 2001. Constituye una unidad ecológica con valores muy importantes, que por sus caracterís- 
ticas físico-geográficas y la cercanía a la zona de convergencia de las brisas, mantiene relaciones desde el punto de vista climático e hidrológico con otros ecosistemas en la provincia, al igual que relaciones biológicas producto principalmente de las biocorrientes establecidas. Tiene una elevada vulnerabilidad al cambio climático y a pesar de las incertidumbres, los pronósticos futuros de la Ciénaga resultan pesimistas, una prueba de ello y se cita de las conclusiones del capítulo de Oceanología del proyecto sobre la adaptación Cuba al cambio climático y que sirvió de base a la Primera Comunicación de Cuba a la Convección Marco de Naciones Unidas para el Cambio Climático, (CMNUCC, 2000: 66) las cayerías interiores de los golfos de Batabanó y Ana María-Guacanayabo, cuyas cotas apenas alcanzan los 0.5 metros, reducirían considerablemente sus extensiones territoriales para el 2050 (ascenso de 21.63 ó 23.30 cm según escenarios de emisiones) y ya para finales del siglo XXI no existirían. Algo similar debería ocurrir con el $60-80 \%$ de la Ciénaga de Zapata y con muchos de los cayos que componen los subarchipiélagos de Los Colorados, Sabana - Camagüey, Canarreos y Jardines de la Reina.

\subsection{Apuntes socio-económicos de la Ciénaga de Zapata}

La Ciénaga constituye por su extensión territorial uno de los municipios mas grandes del país y por su población uno de los menos poblados. Sus principales características socio-económicas son su poca densidad de población, alrededor de 1.99 habitantes por km², la más baja del país y una economía local con muy poco desarrollo.

La población total del municipio es de 8611 hab, de ellos el 51.3\% son hombres. La población en edad laboral es de 5273 hab, aproximadamente el $60 \%$ d e la población. La proporción por sexo alcanza un índice de masculinidad de 106/100, o sea 106 hombres por cada 100 mujeres. El crecimiento de la población es también muy lento y se caracteriza por un equilibrio entre la emigración e inmigración en el territorio y por ende entre la natalidad y la mortalidad. Otro aspecto que llama la atención es que el 34 $\%$ de la población se concentra en dos asentamientos poblacionales Gironcito y Cayo Ramona. La tendencia la dispersión de la población en el territorio que se manifestó en los años 90s fue abruptamente interrumpida y sustituida con una mayor concentración, con la reconstrucción de los asentamientos urbanos tras las afectaciones del huracán Michelle en el año 2001. Este huracán afectó la zona con vientos superiores a los 200 $\mathrm{km} / \mathrm{h}$ y produjo importantes impactos negativos sobre el medioambiente y la esfera socioeconómica.

Las principales ramas económicas son la silvicultura, apicultura, pesca, industria local y turismo. El humedal tiene importantes recursos forestales, que incluyen maderas preciosas. La explotación de estos recursos tiene muy poco valor agregado y la tecnología de corte y extracción son muy atrasadas. Los recursos energéticos principalmente turba y los energéticos forestales (bosques), presentes en el ecosistema no pueden explotarse por la función protectora y/o reguladora que realizan.

Los abundancia de recursos de la pesca (cocodrilos, tortugas, crustáceos, mariscos) son otra de las características de la Ciénaga de Zapata, sin embargo, estos no se explotan adecuadamente. El alto potencial melífero de la ciénaga es explotado principalmente por otros territorios. La producción agrícola es insuficiente y la infraestructura productiva, en particular los viales, resultan deficiente. La producción de artesanía, tiene un valor, relativamente bajo y la actividad turística, aunque creciente, no alcanza aún los niveles que avalan el potencial turístico de la zona. 


\subsection{Apuntes Ecológicos de la Ciénaga de Zapata}

El humedal Ciénaga de Zapata tiene una gran riqueza de la diversidad biológica. Esto es una consecuencia de las buenas condiciones de las funciones ecológicas presentes en la región, así como de la baja asimilación y buen estado natural del territorio. Es una de las regiones con mejor conservación de su estado natural en el país, fundamentalmente por la inaccesibilidad, propia del territorio, y las medidas de protección que se han adoptado desde 1960. En el territorio se encuentran gran cantidad de especies endémicas de la flora y la fauna. 139 plantas endémicas, 14 especies raras, 5 especies endémicas locales, 16 en peligro de extinción, forman parte de esta riqueza. (Alfonso, et al, 2002).

La fragilidad del humedal es una de sus mayores vulnerabilidades al cambio climático. No obstante los ecosistemas tienen buena resiliencia. Esta fragilidad se hace mayor por un incorrecto desempeño de la actividad humana, fundamentalmente, la contaminación, la caza y pesca furtiva y por fenómenos naturales tales como huracanes y el fuego, que en ocasiones tiene causas antropogénicas también.

La tipicidad es otro de los valores ecológicos del ecosistema. Ésta se fundamenta por la presencia de dos manifestaciones geomorfológicas, que sólo se pueden encontrar en este sitio en el país:

1. Los cenotes, que son depresiones cubiertas de agua de aproximadamente igual profundidad que aparecen sobre superficies cársicas.

2. Los petenes, que son cayos de manglares en medio de los herbazales de ciénaga en cuyo centro, generalmente, aparece un lago.

\subsection{Apuntes sobre el clima en la Ciénaga de Zapata}

El clima de la ciénaga ha mostrado alguna variabilidad en los últimos diez años. En la década de los años 90 del siglo XX la temperatura media creció respecto a la 61-80. Este crecimiento se registro también en las temperaturas máximas y mínimas. En esta última variable el aumento experimentado fue mayor que en las máximas. Las precipitaciones han mostrado una ligera variación en los últimos diez años. La ciénaga ha sido afectada directamente por dos huracanes en los últimos siete años. El último de ellos, el citado huracán Michelle, es el organismo tropical más intenso que ha afectado la provincia en los últimos cincuenta años (Moya, 2002).

Existe una gran incertidumbre sobre el comportamiento futuro del clima, fundamentalmente por el desconocimiento que se tiene de la respuesta que dará la atmósfera al calentamiento global, sin embargo algunas interrogantes aparecen ya y en muchos casos la comunidad científica le atribuye alguna probabilidad de ocurrencia.

¿Ocurrirán cambios en el comportamiento de las precipitaciones?

¿Se harán más frecuentes e intensas las sequías?

¿Aumentarán los eventos de grandes precipitaciones?

¿Habrá una mayor actividad de huracanes?

¿Estará la ciénaga expuesta a tormentas extratropicales?

¿La temperatura aumentará?

¿Aumentará el nivel del mar? 
Tabla 1

AUMENTO DEL NIVEL MEDIO DEL MAR ESPERADO SEGÚN KYOTO 1

\begin{tabular}{|c|c|}
\hline Año & $\begin{array}{c}\text { Aum. NMM } \\
(\mathrm{cm})\end{array}$ \\
\hline 2010 & 4.71 \\
\hline 2030 & 11.91 \\
\hline 2050 & 21.63 \\
\hline 2100 & 50.82 \\
\hline
\end{tabular}

Fuente Primera Comunicación de Cuba a la CMNUCC.

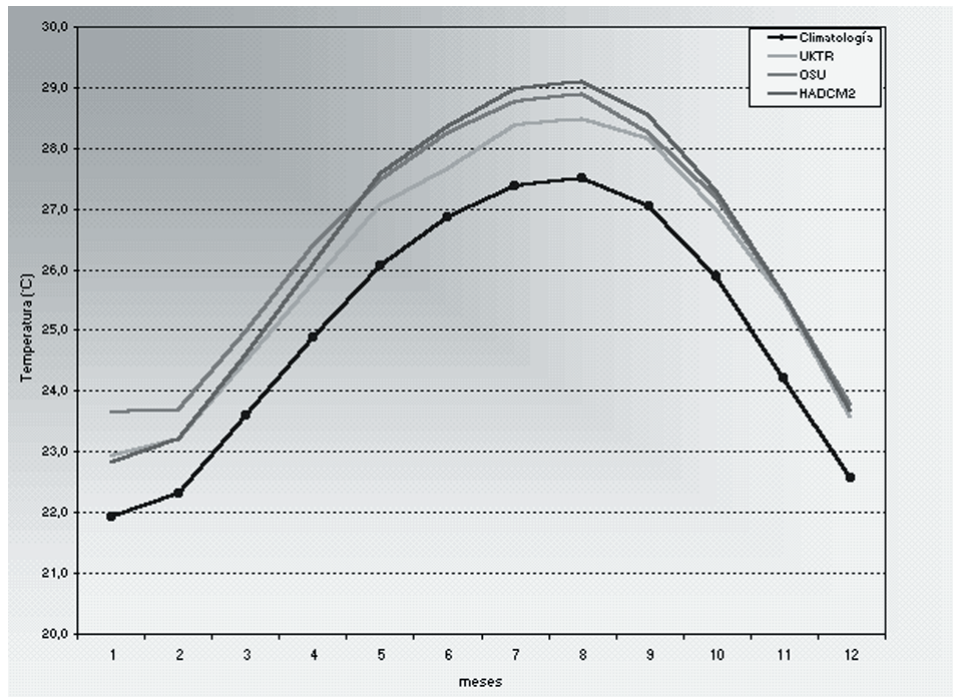

FIgURA 1. Aumento de la temperatura para el 2050 respecto a 1990. Fuente: Primera comunicación de Cuba al CMNUCC. 
Estudios del Centro Nacional del Clima muestran, que para la rejilla donde se ubica la ciénaga una serie de variaciones son esperadas para diferentes escenarios climáticos algunos ejemplos de estas se citan a continuación.

\subsection{Vulnerabilidades, Impactos y Adaptación}

La Ciénaga de Zapata es muy vulnerable al cambio climático y ya se ha citado que para fines del siglo actual pudiera desaparecer una considerable extensión de la misma. Por otra parte una serie de impactos potenciales por cambio climático pueden afectar el territorio y provocará daños sensibles al ecosistema y a la actividad socioeconómica que en el mismo se desarrolla.

\subsubsection{Vulnerabilidad}

Entre las principales vulnerabilidades e impactos potenciales cabe destacar:

Vulnerabilidades:

1. Poca altura sobre el nivel del mar.

2. Asentamientos humanos en zonas expuestas a la penetración del mar.

3. Exposición de las fuentes de abastos a la intrusión salina.

4. Explotación de recursos naturales.

5. Acción de predadores furtivos.

6. La exposición, endemismo local y fragilidad de la flora y fauna.

7. Explotación del humedal y fragmentación de ecosistemas.

8. Fragilidad de los ecosistemas.

9. Exposición a tormentas y fenómenos severos del tiempo.

10. Incertidumbres en las respuestas de la atmósfera e impactos por cambio climático.

11. No existe una estrategia de adaptación y mitigación de impactos por variabilidad y cambio climático.

\subsubsection{Impactos}

Entre los principales impactos por cambio climático, que se producirán en la Ciénaga encontramos:

a) Afectación en las funciones ecológicas del ecosistema.

b) Perdidas de hábitat.

c) Afectaciones a la fuente de abasto de agua por inclusión salina.

d) Aumento de la erosión costera.

e) Afectaciones a la flora y fauna.

f) Pérdidas por inundaciones, fenómenos meteorológicos extremos e incendios.

g) Afectaciones al transporte de sedimentos y nutrientes.

h) Variaciones en los patrones epidemiológicos y de las epifítias fundamentalmente por cambios en las condiciones medioambientales donde se desarrollan los patógenos.

i) Incertidumbres sobre que hacer ante el cambio climático.

En la siguiente tabla se muestra la debilidad de las vulnerabilidades, frente al potencial de los impactos, mostrando cuales son las vulnerabilidades, naturales o asociadas a la antropogenización que tienen mayor exposición a los impactos y el potencial de afectación de los impactos para las diferentes vulnerabilidades o acciones de vulnerabilidad. 
Tabla 2

POTENCIAL DE VULNERABILIDAD E IMPACTOS PARA LA CIÉNAGA DE ZAPATA.

\begin{tabular}{|l|c|c|c|c|c|c|c|c|c|c|}
\hline & $\mathbf{a}$ & $\mathbf{b}$ & $\mathbf{c}$ & $\mathbf{d}$ & $\mathbf{e}$ & $\mathbf{f}$ & $\mathbf{g}$ & $\mathbf{h}$ & $\mathbf{i}$ & Debilidad \\
\hline 1 & & $\mathrm{X}$ & $\mathrm{X}$ & $\mathrm{X}$ & $\mathrm{X}$ & $\mathrm{X}$ & & & $\mathrm{X}$ & 6 \\
\hline 2 & & $\mathrm{X}$ & $\mathrm{X}$ & $\mathrm{X}$ & $\mathrm{X}$ & $\mathrm{X}$ & $\mathrm{X}$ & $\mathrm{X}$ & $\mathrm{X}$ & 8 \\
\hline 3 & & & $\mathrm{X}$ & $\mathrm{X}$ & & $\mathrm{X}$ & & & $\mathrm{X}$ & 4 \\
\hline 4 & $\mathrm{X}$ & $\mathrm{X}$ & $\mathrm{X}$ & & $\mathrm{X}$ & $\mathrm{X}$ & $\mathrm{X}$ & & $\mathrm{X}$ & 7 \\
\hline 5 & & $\mathrm{X}$ & & & $\mathrm{X}$ & & & & $\mathrm{X}$ & 3 \\
\hline 6 & $\mathrm{X}$ & $\mathrm{X}$ & & $\mathrm{X}$ & $\mathrm{X}$ & $\mathrm{X}$ & & & $\mathrm{X}$ & 6 \\
\hline 7 & & $\mathrm{X}$ & & $\mathrm{X}$ & $\mathrm{X}$ & $\mathrm{X}$ & $\mathrm{X}$ & & $\mathrm{X}$ & 6 \\
\hline 8 & $\mathrm{X}$ & $\mathrm{X}$ & & $\mathrm{X}$ & $\mathrm{X}$ & $\mathrm{X}$ & & & $\mathrm{X}$ & 6 \\
\hline 9 & & $\mathrm{X}$ & & $\mathrm{X}$ & $\mathrm{X}$ & $\mathrm{X}$ & $\mathrm{X}$ & & $\mathrm{X}$ & 6 \\
\hline 10 & $\mathrm{X}$ & $\mathrm{X}$ & $\mathrm{X}$ & $\mathrm{X}$ & $\mathrm{X}$ & $\mathrm{X}$ & $\mathrm{X}$ & $\mathrm{X}$ & $\mathrm{X}$ & 9 \\
\hline 11 & $\mathrm{X}$ & $\mathrm{X}$ & $\mathrm{X}$ & $\mathrm{X}$ & $\mathrm{X}$ & $\mathrm{X}$ & $\mathrm{X}$ & $\mathrm{X}$ & $\mathrm{X}$ & 9 \\
\hline Afectacion & 5 & 10 & 6 & 9 & 10 & 10 & 6 & 3 & 11 & \\
\hline
\end{tabular}

\subsubsection{Adaptación}

Si se tiene en cuenta lo anterior y se analiza relación e entre vulnerabilidad e impactos, una serie de medidas de adaptación al cambio climático se determinan ya, aunque es necesario desarrollar un estudio más profundo que permita una mejor adaptación del ecosistema a los escenarios futuros. Evaluando las vulnerabilidades e impactos para diferentes escenarios. Algunas de las medidas se mencionan a continuación:

- Desarrollo de una estrategia de adaptación con la participación de investigadores, especialistas y administradores del humedal.

- Enriquecimiento de los programas y planes de manejo con medidas de adaptación y mitigación al cambio climático, teniendo en cuanta no sólo la vulnerabilidad del ecosistema, sino el papel que juega también en la mitigación del cambio climático y sus impactos, como sumidero de $\mathrm{CO}_{2}$ y primera barrera ante los eventos extremos del tiempo y clima.

- Restauración y rehabilitamiento de zonas afectadas, eliminando el stress y disminuyendo su vulnerabilidad.

- Evitar nuevas presiones que afecten la capacidad de respuesta y recuperación.

- Evitar la fragmentación de los ecosistemas.

- Protección y disminución de población en los lugares más bajos y cercanos a la costa.

- Disminución de vulnerabilidad en la infraestructura constructiva.

- Monitoreo ecológico y climático.

- Inclusión del tema del CC en la actividad de educación ambiental que se lleva en el humedal.

- Continuar con la política de reforestación de arboles y manglares. 
Como resultado de estas medidas de adaptación se propone un proyecto, dirigido a establecer una estrategia de adaptación en Ciénaga de Zapata y Majaguillar, en el primer caso ya se conoce su importancia y en el segundo es uno de los más importantes de la provincia y destaca respecto a la ciénaga en su ubicación en la costa norte.

\section{CONCLUSIONES}

La relación de la Ciénaga de Zapata con el cambio climático, debe verse desde sus aristas, la primera, como mitigador del calentamiento y de los impactos del cambio al tener entre sus funciones la de sumidero de $\mathrm{CO}_{2}$ y constituir la primera barrera ante los fenómenos meteorológicos extremos y la segunda arista, referida a su vulnerabilidad a los escenarios de cambio.

La Ciénaga presenta grandes extensiones de masa verde que constituye sumidero de $\mathrm{CO}_{2}$ y su potencial de absorción de este gas de efecto invernadero es de las más elevada en el territorio provincial, por otra parte las extensas áreas despobladas constituyen una barrera a las inclemencias de tormentas tropicales y otros eventos meteorológicos extremos.

La Ciénaga de Zapata es un sitio altamente vulnerable al cambio climático y a la acción de fenómenos severos extremos. La baja altura sobre el nivel del mar, su cercanía a la costa, la fragilidad de sus ecosistemas, el endemismo de su flora y fauna, entre otras son vulnerabilidades del territorio, cuyas riquezas ecológicas y económicas, amenazan con desaparecer por la acción del cambio climático.

Las incertidumbres respecto al cambio climático y el no contar con este tópico dentro de los planes de manejos son otros aspectos que hacen más vulnerable el territorio. Esto realza la necesidad de una política de adaptación con el objetivo de disminuir las pérdidas y facilitar la adaptación del mayor humedal del Caribe insular a los escenarios climáticos futuros.

\section{BIBLIOGRAFÍA}

1. Alfonso A. et al (2002) Informe final del Proyecto Territorial Desarrollo de un sistema de Manejo del Humedal Cienaga de Zapata. pp. 167.

2. Centelles A. (1999) Conferencia Cambio Climática e Impactos. Reunión Nacional de Clima. Ciego de Ávila.

3. Gutiérrez T. y otros (2000) Impactos del Cambio Climático y Medidas de Adaptación en Cuba. C. Habana. pp. 24-49.

4. IPCC (1995) The regional impacts of Climate Change. An Assessment of Vulnerability. IPCC, WMO, UNEP. Cambridge University Press 1. pp. 1001.

5. IPCC (2001). IPCC 2001. Impacts, Adaptation and Vulnerability. IPCC, WMO, UNEP. Cambridge University Press. pp. 1009.

6. Moya B. (2002). Climate Change in Varadero one of the most important touristic places in Caribbean sea. IHDP. Workshop 2002 Bonn. Alemania pp. 3-7.

7. Moya B. (2004) Informe parcial del Proyecto Territorial Cambio Climático en Varadero. Vulnerabilidad, impactos y adaptación. CITMA. Matanzas. Cuba. Pp. 52.

8. RAMSAR Los humedales y el cambio climático. Examen de la colaboración entre la Convención sobre los Humedales (RAMSAR, Irán 1971) y la Convención Marco de las Naciones Unidas sobre el Cambio Climático. 1999 www.ramsar.org/key_unfccc_bkgd.htm

9. RAMSAR Notas informativas sobre los valores y las funciones de los humedales. Mitigación del cambio climático. Hoja informativa N`5 2000 www.ramsar.org/values_climate_s.htm

10. RAMSAR $8^{\text {a }}$ Reunión de la Conferencia de las Partes Contratantes en la Convención sobre los Humedales (Ramsar, Irán,1971) Valencia, España, 18 al 26 de Noviembre de 2002 pp. 4. 